\title{
MODULES WITH FI-EXTENDING HULLS
}

\author{
GARY F. BIRKENMEIER \\ Department of Mathematics, University of Louisiana at Lafayette, Lafayette, LA 70504-1010, USA \\ e-mail:gfb1127@louisiana.edu \\ JAE KEOL PARK \\ Department of Mathematics, Busan National University, Busan 609-735, South Korea \\ e-mail: jkpark@pusan.ac.kr \\ and S. TARIQ RIZVI \\ Department of Mathematics, Ohio State University, Lima, OH 45804-3576, USA \\ e-mail:rizvi.1@osu.edu
}

(Received 20 December 2007; accepted 17 October 2008)

\begin{abstract}
It is shown that every finitely generated projective module $P_{R}$ over a semiprime ring $R$ has the smallest FI-extending essential module extension $H_{\mathfrak{F} \mathfrak{I}}\left(P_{R}\right)$ (called the absolute FI-extending hull of $\left.P_{R}\right)$ in a fixed injective hull of $P_{R}$. This module hull is explicitly described. It is proved that $\widehat{Q}_{\mathfrak{F} \mathfrak{I}}\left(\operatorname{End}\left(P_{R}\right)\right) \cong \operatorname{End}\left(H_{\mathfrak{F} \mathfrak{I}}\left(P_{R}\right)\right)$, where $\widehat{Q}_{\mathfrak{F} \mathfrak{I}}\left(\operatorname{End}\left(P_{R}\right)\right)$ is the smallest right FI-extending right ring of quotients of $\operatorname{End}\left(P_{R}\right)$ (in a fixed maximal right ring of quotients of $\operatorname{End}\left(P_{R}\right)$ ). Moreover, we show that a finitely generated projective module $P_{R}$ over a semiprime ring $R$ is FI-extending if and only if it is a quasi-Baer module and if and only if $\operatorname{End}\left(P_{R}\right)$ is a quasi-Baer ring. An application of this result to $C^{*}$-algebras is considered. Various examples which illustrate and delimit the results of this paper are provided.
\end{abstract} $16 \mathrm{~S} 50$.

2000 Mathematics Subject Classification. Primary 16N60, 16D40; Secondary

An important technique used to study an algebraic object is to search for an overobject that has the following properties: (1) it belongs to a class $\mathfrak{K}$ with some desirable properties; (2) it is explicitly computable; (3) information can be transferred between the base object and its overobject (thus one tries to find an overobject from $\mathfrak{K}$ which is 'close to' the base object).

In module theory the class of injective modules and, its generalization, the class of extending modules have the property that every submodule of a member is essential in a direct summand of the member. This property, originated by Chatters and Hajarnavis in [21], ensures a rich structure theory for these classes. Although every module has an injective hull, it is usually hard to compute. For many modules a minimal essential extension which belongs to the class of extending modules may not exist (e.g. $\oplus_{n=1}^{\infty} \mathbb{Z} \mathbb{Z}$, see comment above Proposition 8). Moreover the class of extending modules lacks some important closure properties (e.g. it is not closed under direct sums).

Throughout this paper all rings are associative with identity and $R$ denotes such a ring. All modules are unitary. Recall from [12] that a right $R$-module $M_{R}$ is $F I$ extending if every fully invariant submodule of $M_{R}$ is essential in a direct summand of $M_{R}$. A ring $R$ is right FI-extending if $R_{R}$ is FI-extending. Note that the set of fully 
invariant submodules of a module $M_{R}$ includes the socle, Jacobson radical, torsion submodule for a torsion theory (e.g., $Z\left(M_{R}\right)$, the singular submodule), and $M I$ for all right ideals $I$ of $R$, etc. Hence, the FI-extending condition provides an 'economical use' of the extending condition by targeting only the fully invariant submodules, and thus some of the most important submodules of $M_{R}$ for an essential splitting of $M_{R}$. Natural examples of FI-extending rings and modules abound: direct sums of uniform modules, more specifically all finitely generated Abelian groups, semisimple modules, prime rings, serial rings, semiprime right finitely pseudo-Frobenius (FPF) rings. Also it is shown in [13, Corollary 1.9] that semiprime right Noetherian group algebras over a field are right FI-extending. Note that in this case if the group is Abelian, then the group algebra is extending.

In [19] we showed that every semiprime ring $R$ has the smallest right FI-extending right ring of quotients $\widehat{Q}_{\mathfrak{F} \mathfrak{I}}(R)$. In this paper, we further develop the FI-extending concept by showing that over a semiprime ring $R$, every finitely generated projective module $P_{R}$ has the smallest FI-extending essential extension $H_{\mathfrak{F} \mathfrak{I}}\left(P_{R}\right)$ (called the absolute FI-extending hull of $\left.P_{R}\right)$ in a fixed injective hull of $P_{R}$. Moreover, $H_{\mathfrak{F} \mathfrak{I}}\left(P_{R}\right)$ is easily computable (see Theorem 6 and Proposition 8), it is from a class for which direct sums and direct summands are FI-extending, and since $H_{\mathfrak{F} \mathfrak{I}}\left(P_{R}\right)$ is finitely generated and projective over $\widehat{Q}_{\mathfrak{F} \mathfrak{I}}(R)$, we are assured of a reasonable transfer of information between $P_{R}$ and $H_{\mathfrak{F} \mathfrak{I}}\left(P_{R}\right)$ (e.g. see Theorem 12 and Corollary 13).

Since many well-known types of Banach algebras are semiprime (e.g. $C^{*}$-algebras), our results are applicable. Finitely generated modules over a Banach algebra are considered in [26]. Kaplansky [28] defined $A W^{*}$-modules over a $C^{*}$-algebra and used them to answer several questions concerning automorphisms and derivations on certain types of $C^{*}$-algebras. Furthermore work using these modules appeared in [6]. Moreover, from [20, p. 352], every algebraically finitely generated $C^{*}$-module $M$ is projective, hence $H_{\mathfrak{F} \mathfrak{I}}(M)$ exists. Since every $C^{*}$-algebra $A$ is both semiprime and nonsingular, $\widehat{Q}_{\mathfrak{F} \mathfrak{I}}(A)$ always exists by [19]. Also in [19], we characterized all $C^{*}$-algebras with only finitely many minimal prime ideals and showed that for such $A, \widehat{Q}_{\mathfrak{F} \mathfrak{I}}(A)$ is also a $C^{*}$-algebra. Thus our results should yield fruitful applications to projective modules over $C^{*}$-algebras, as well as many other algebras in Functional Analysis.

According to [15] a module $M_{R}$ is called strongly FI-extending if every fully invariant submodule of $M_{R}$ is essential in a fully invariant direct summand of $M_{R}$ (e.g. a non-singular FI-extending module). Note that the class of strongly FI-extending modules is closed under direct summands and is contained in the class of FI-extending modules. A ring $R$ is said to be right strongly FI-extending if $R_{R}$ is strongly FI-extending. We use $\mathfrak{F} \mathfrak{I}$ (resp., $\mathfrak{E}, \mathfrak{S} \mathfrak{F} \mathfrak{I}$ ) to denote the class of FI-extending (resp., extending, strongly FI-extending) right modules or the class of right FI-extending (resp., right extending, right strongly FI-extending) rings according to the context (see [7-10, 12, 13 and 33] for details and examples of the (strongly) FI-extending property of modules and rings, also see [21] and [23] for the extending property of modules and rings).

If $N_{R}$ is a submodule of $M_{R}$, then $N_{R}$ is essential (resp., dense also called rational) in $M_{R}$ if for any $0 \neq x \in M$, there exists $r \in R$ such that $0 \neq x r \in N$ (resp., for any $x, y \in M$ with $0 \neq x$, there exists $r \in R$ such that $x r \neq 0$, and $y r \in N)$. For $R$-modules $M_{R}$ and $N_{R}$, we use $N_{R} \leq M_{R}, N_{R} \leq{ }^{\text {ess }} M_{R}$ and $N_{R} \unlhd M_{R}$ to denote that $N_{R}$ is a submodule of $M_{R}, N_{R}$ is an essential submodule of $M_{R}$ and $N_{R}$ is a fully invariant submodule of $M_{R}$, respectively. Let $E\left(M_{R}\right)$ denote an injective hull of a module $M_{R}$. Recall that a right ring of quotients $T$ of a ring $R$ is an overring of $R$ such that $R_{R}$ is dense in $T_{R}$. An overring $S$ of a ring $R$ is called a right essential overring of $R$ if $R_{R}$ is essential in $S_{R}$. For a ring $R, \mathbf{J}(R), \mathbf{B}(R)$, $\operatorname{Mat}_{n}(R)$ and $Q(R)$ denote the Jacobson 
radical of $R$, the set of central idempotents of $R$, the $n \times n$ matrix ring over $R$ and the maximal right ring of quotients of $R$, respectively.

Definition 1. We fix an injective hull $E\left(M_{R}\right)$ of $M_{R}$ and a maximal right ring of quotients $Q(R)$ of $R$.

(i) Let $\mathfrak{M}$ be a class of right $R$-modules and $M_{R}$ a right $R$-module. We call, when it exists, a module $H_{\mathfrak{M}}\left(M_{R}\right)$ the absolute $\mathfrak{M}$ hull of $M_{R}$ if $H_{\mathfrak{M}}\left(M_{R}\right)$ is the smallest essential extension of $M_{R}$ in $E\left(M_{R}\right)$ that belongs to $\mathfrak{M}$.

(ii) Let $\mathfrak{K}$ be a class of rings and $R$ a ring. We call, when it exists, a ring $\widehat{Q}_{\mathfrak{K}}(R)$ the $\mathfrak{K}$ absolute to $Q(R)$ right ring hull of $R$ if $\widehat{Q}_{\mathfrak{K}}(R)$ is the smallest right ring of quotients of $R$ in $Q(R)$ that belongs to $\mathfrak{K}$.

LEMMA 2 ([17, Lemma 1.4]). Let $T$ be a right ring of quotients of a ring $R$.

(i) For right ideals $X$ and $Y$ of $T$, if $X_{T} \leq{ }^{\text {ess }} Y_{T}$, then $X_{R} \leq{ }^{\text {ess }} Y_{R}$.

(ii) If $X_{R} \unlhd T_{R}$, then $X_{R} \leq^{\text {ess }} T X T_{R}$.

We recall from $[\mathbf{2 2}, \mathbf{2 9}, \mathbf{3 6}]$ that a ring $R$ is called quasi-Baer (resp., Baer) if the right annihilator of every right ideal (resp., non-empty subset) of $R$ is generated, as a right ideal, by an idempotent (see $[\mathbf{1 1}, \mathbf{2 2}, \mathbf{3 6}]$ for more on quasi-Baer rings). The class of quasi-Baer rings is denoted by $\mathfrak{q} \mathfrak{B}$. Mewborn [32] showed the existence of a Baer absolute to $Q(R)$ ring hull for a commutative semiprime ring $R$ (which, in this case, coincides with $\widehat{Q}_{\mathfrak{q} \mathfrak{B}}(R)$ ). For more details on $\widehat{Q}_{\mathfrak{q} \mathfrak{B}}(R)$ and its applications, see [19].

Lemma $3\left(\left[19\right.\right.$, Theorem 3.3]). Assume that $R$ is a semiprime ring. Then $\widehat{Q}_{\mathfrak{F} \mathfrak{I}}(R)=$ $R \mathbf{B}(Q(R))=\widehat{Q}_{\mathfrak{q} \mathfrak{B}}(R)$, where $R \mathbf{B}(Q(R))$ is the subring of $Q(R)$ generated by $R$ and $\mathbf{B}(Q(R))$.

LEMMA 4. Let $T$ be a right ring of quotients of a ring $R$. Then $\operatorname{End}\left(T_{R}\right)=$ $\operatorname{End}\left(T_{T}\right) \cong T$.

Proof. See [31, p. 94] for the proof.

LEMMA 5. If $M_{R}$ is a FI-extending module, then $f M \subseteq M$ for any central idempotent $f$ of $\operatorname{End}\left(E\left(M_{R}\right)\right)$.

Proof. Let $f \in \mathbf{B}\left(\operatorname{End}\left(E\left(M_{R}\right)\right)\right)$. Then $f E\left(M_{R}\right) \cap M \unlhd M_{R}$. Since $M_{R}$ is FIextending, there is $g=g^{2} \in \operatorname{End}\left(M_{R}\right)$ such that $f E\left(M_{R}\right) \cap M_{R} \leq^{\text {ess }} g M_{R} \leq^{\text {ess }} \bar{g} E\left(M_{R}\right)$, where $\bar{g}$ is the projection from $E\left(M_{R}\right)=E\left(g M_{R}\right) \oplus E\left((1-g) M_{R}\right)$ to $E\left(g M_{R}\right)$. Note that $f E\left(M_{R}\right) \cap M_{R} \leq{ }^{\text {ess }} f E\left(M_{R}\right)$. Thus $f=\bar{g}$ since $f$ is a central idempotent in $\operatorname{End}\left(E\left(M_{R}\right)\right)$. Therefore $f M=\bar{g} M=g M \subseteq M$.

The converse of Lemma 5 does not hold. For this, see $R_{R}$ in Example 9. Observe that when $R$ is semiprime $\operatorname{End}\left(\oplus^{n} R_{R}\right) \cong \operatorname{Mat}_{n}(R) \subseteq \operatorname{Mat}_{n}\left(\widehat{Q}_{\mathfrak{F} \mathfrak{I}}(R)\right) \cong$ $\operatorname{End}\left(\oplus^{n} \widehat{Q}_{\mathfrak{F} \mathfrak{I}}(R)_{\widehat{Q}_{\mathfrak{F}}(R)}\right)=\operatorname{End}\left(\oplus^{n} \widehat{Q}_{\mathfrak{F} \mathfrak{I}}(R)_{R}\right)$ by Lemma 4 . Thus, in the sequel, we identify $\operatorname{End}\left(\oplus^{n} R_{R}\right)$ as a subring of $\operatorname{End}\left(\oplus^{n} \widehat{Q}_{\mathfrak{F} \mathfrak{I}}(R)_{\widehat{Q}_{\mathfrak{F} \mathfrak{I}}(R)}\right)=\operatorname{End}\left(\oplus^{n} \widehat{Q}_{\mathfrak{F} \mathfrak{I}}(R)_{R}\right)$.

We first show the existence of the absolute FI-extending hull for every finitely generated projective module over a semiprime ring. Also this module hull is explicitly described.

THEOREM 6. Every finitely generated projective module $P_{R}$ over a semiprime ring $R$ has the absolute FI-extending hull $H_{\mathfrak{F} \mathfrak{I}}\left(P_{R}\right)$. Explicitly, $H_{\mathfrak{F} \mathfrak{I}}\left(P_{R}\right) \cong e\left(\oplus^{n} \widehat{Q}_{\mathfrak{F} \mathfrak{I}}(R)_{R}\right)$ where $P \cong e\left(\oplus^{n} R_{R}\right)$, for some $n$ and $e=e^{2} \in \operatorname{End}\left(\oplus^{n} R_{R}\right)$.

Proof. Step 1. $\widehat{Q}_{\mathfrak{F} \mathfrak{I}}(R)_{R}$ is strongly FI-extending. 
Proof of Step 1. Let $T=\widehat{Q}_{\mathfrak{F} \mathfrak{I}}(R)$. Then $T=R \mathbf{B}(Q(R))$ (by Lemma 3 ) is semiprime and quasi-Baer. To show that $T_{R}$ is strongly FI-extending, let $X_{R} \unlhd T_{R}$. By Lemma 2(ii), $X_{R} \leq{ }^{\text {ess }} T X T_{R}$. From [12, Theorem 4.7], there exists $c \in \mathbf{B}(T)$ such that $T X T_{T} \leq^{\text {ess }}$ $c T_{T}$. By Lemma 2(i), $T X T_{R} \leq{ }^{\text {ess }} c T_{R}$. By Lemma $4, \operatorname{End}\left(T_{R}\right)=\operatorname{End}\left(T_{T}\right) \cong T$. Hence for any $\lambda \in \operatorname{End}\left(T_{R}\right), \lambda(c T)=c(\lambda T)$. Thus $c T_{R} \unlhd T_{R}$, so $T_{R}$ is strongly FI-extending as $X_{R} \leq{ }^{\text {ess }} c T_{R}$.

Step 2. $H_{\mathfrak{F} \mathfrak{I}}\left(\oplus^{n} R_{R}\right)=\oplus^{n} \widehat{Q}_{\mathfrak{F} \mathfrak{I}}(R)_{R}$.

Proof of Step 2. Note that $\widehat{Q}_{\mathfrak{F} \mathfrak{I}}(R)_{R}$ is FI-extending by Step 1 , so $\oplus^{n} \widehat{Q}_{\mathfrak{F} \mathfrak{I}}(R)_{R}$ is FI-extending [12, Theorem 1.3]. Suppose that $N_{R}$ is FI-extending with $\oplus^{n} R_{R} \leq N_{R} \leq$ $E\left(\oplus^{n} R_{R}\right)=\oplus^{n} E\left(R_{R}\right)$. Note that $\mathbf{B}(Q(R))=\mathbf{B}\left(\operatorname{End}\left(E\left(R_{R}\right)\right)\right.$. Take $f \in \mathbf{B}(Q(R))$. Let $f I_{n}$ be the $n$-by- $n$ diagonal matrix with $f$ on the diagonal, where $I_{n}$ is the identity matrix in $\operatorname{End}\left(\oplus^{n} E\left(R_{R}\right)\right) \cong \operatorname{Mat}_{n}\left(\operatorname{End}\left(E\left(R_{R}\right)\right)\right)$. Then $f I_{n} \in \mathbf{B}\left(\operatorname{End}\left(\oplus^{n} E\left(R_{R}\right)\right)\right)$. Thus by Lemma 5,

$$
f I_{n} N \subseteq N, \quad \text { so } \quad f I_{n}\left(\begin{array}{c}
R \\
\vdots \\
R
\end{array}\right) \subseteq N, \quad \text { where } \quad\left(\begin{array}{c}
R \\
\vdots \\
R
\end{array}\right)=\oplus^{n} R_{R}
$$

Observe that $\widehat{Q}_{\mathfrak{F} \mathfrak{I}}(R)=R \mathbf{B}(Q(R))$ from Lemma 3 . Therefore $\oplus^{n} \widehat{Q}_{\mathfrak{F} \mathfrak{I}}(R)_{R} \leq N_{R}$, hence $H_{\mathfrak{F} \mathfrak{I}}\left(\oplus^{n} R_{R}\right)=\oplus^{n} \widehat{Q}_{\mathfrak{F} \mathfrak{I}}(R)_{R}$.

Step 3. $H_{\mathfrak{F} \mathfrak{I}}\left(e\left(\oplus^{n} R_{R}\right)\right)=e\left(\oplus^{n} \widehat{Q}_{\mathfrak{F} \mathfrak{I}}(R)_{R}\right)$.

Proof of Step 3. We may assume that $P_{R}=e\left(\oplus^{n} R_{R}\right)$. Therefore, $\oplus^{n} \widehat{Q}_{\mathfrak{F} \mathfrak{I}}(R)_{R}=$ $e\left(\oplus^{n} \widehat{Q}_{\mathfrak{F} \mathfrak{I}}(R)_{R}\right) \oplus(1-e)\left(\oplus^{n} \widehat{Q}_{\mathfrak{F} \mathfrak{I}}(R)_{R}\right)$. Since $\widehat{Q}_{\mathfrak{F} \mathfrak{I}}(R)_{R}$ is strongly FI-extending by Step $1, \oplus^{n} \widehat{Q}_{\mathfrak{F} \mathfrak{I}}(R)_{R}$ is strongly FI-extending by [15, Theorem 3.3]. Hence $e\left(\oplus^{n} \widehat{Q}_{\mathfrak{F} \mathfrak{I}}(R)_{R}\right)$ is strongly FI-extending [15, Theorem 2.4]. Let $N_{R}$ be FI-extending such that $e\left(\oplus^{n} R_{R}\right) \leq$ $N_{R} \leq E\left(e\left(\oplus^{n} R_{R}\right)\right)$. Then $\oplus^{n} R_{R}=e\left(\oplus^{n} R_{R}\right) \oplus(1-e)\left(\oplus^{n} R_{R}\right) \leq N_{R} \oplus(1-e)\left(\oplus^{n} R_{R}\right) \leq$ $N_{R} \oplus E\left[(1-e)\left(\oplus^{n} R_{R}\right)\right]$. Now since $N_{R}$ is FI-extending and $E\left[(1-e)\left(\oplus^{n} R_{R}\right)\right]$ is injective, $N_{R} \oplus E\left[(1-e)\left(\oplus^{n} R_{R}\right)\right]$ is FI-extending [12, Theorem 1.3]. Hence, by Step $2, H_{\mathfrak{F} \mathfrak{I}}\left(\oplus^{n} R_{R}\right)=\oplus^{n} \widehat{Q}_{\mathfrak{F} \mathfrak{I}}(R)_{R} \leq N_{R} \oplus E\left[(1-e)\left(\oplus^{n} R_{R}\right)\right]$.

To prove that $e\left(\oplus^{n} \widehat{Q}_{\mathfrak{F} \mathfrak{I}}(R)_{R}\right) \leq N_{R}$, take $e \alpha \in e\left(\oplus^{n} \widehat{Q}_{\mathfrak{F} \mathfrak{I}}(R)_{R}\right)$ with $\alpha \in \oplus^{n} \widehat{Q}_{\mathfrak{F} \mathfrak{I}}(R)_{R}$. Since $e\left(\oplus^{n} \widehat{Q}_{\mathfrak{F} \mathfrak{I}}(R)_{R}\right) \leq N_{R} \oplus E\left[(1-e)\left(\oplus^{n} R_{R}\right)\right], e \alpha=n+y$ for some $n \in N$ and $y \in$ $E\left[(1-e)\left(\oplus^{n} R_{R}\right)\right]$. Thus

$$
e \alpha-n=y \in\left[e\left(\oplus^{n} \widehat{Q}_{\mathfrak{F} \mathfrak{I}}(R)_{R}\right)+N\right] \cap E\left[(1-e)\left(\oplus^{n} R_{R}\right)\right] .
$$

Note that $E\left[e\left(\oplus^{n} \widehat{Q}_{\mathfrak{F} \mathfrak{I}}(R)_{R}\right)\right]=E\left[e\left(\oplus^{n} R_{R}\right)\right]$ because $e\left(\oplus^{n} R_{R}\right) \leq^{\text {ess }} e\left(\oplus^{n} \widehat{Q}_{\mathfrak{F} \mathfrak{I}}(R)_{R}\right)$. So $\quad\left[e\left(\oplus^{n} \widehat{Q}_{\mathfrak{F} \mathfrak{I}}(R)_{R}\right)+N\right] \cap E\left((1-e)\left(\oplus^{n} R_{R}\right)\right] \leq E\left[e\left(\oplus^{n} R_{R}\right)\right] \cap E\left[(1-e)\left(\oplus^{n} R_{R}\right)\right]=0$. Hence $e \alpha-n=y=0$, so $e \alpha=n \in N$. Therefore $e\left(\oplus^{n} \widehat{Q}_{\mathfrak{F} \mathfrak{I}}(R)_{R}\right) \leq N_{R}$. Consequently, $H_{\mathfrak{F} \mathfrak{I}}\left(e\left(\oplus^{n} R_{R}\right)\right)=e\left(\oplus^{n} \widehat{Q}_{\mathfrak{F} \mathfrak{I}}(R)_{R}\right)$.

Step 4. $H_{\mathfrak{F} \mathfrak{I}}\left(P_{R}\right) \cong e\left(\oplus^{n} \widehat{Q}_{\mathfrak{F} \mathfrak{I}}(R)_{R}\right)$.

Proof of Step 4. Let $\sigma: P_{R} \rightarrow e\left(\oplus^{n} R_{R}\right)$ be an isomorphism. Then $\sigma$ can be extended to an isomorphism $\bar{\sigma}: E\left(P_{R}\right) \rightarrow E\left(e\left(\oplus^{n} R_{R}\right)\right)$. It is easy to check that $H_{\mathfrak{F} \mathfrak{I}}\left(P_{R}\right)=\bar{\sigma}^{-1}\left(e\left(\oplus^{n} \widehat{Q}_{\mathfrak{F} \mathfrak{I}}(R)_{R}\right)\right) \cong e\left(\oplus^{n} \widehat{Q}_{\mathfrak{F} \mathfrak{I}}(R)_{R}\right)$.

REMARK. From the proof of Theorem 6, we see that the absolute strongly FI-extending hull and the absolute FI-extending hull of a finitely generated projective module $P_{R}$ 
over a semiprime ring $R$ coincide (hence both direct sums and direct summands of $H_{\mathfrak{F} \mathfrak{I}}\left(P_{R}\right)$ are FI-extending). Thus $H_{\mathfrak{S F} \mathfrak{I}}\left(P_{R}\right) \cong e\left(\oplus^{n} \widehat{Q}_{\mathfrak{F} \mathfrak{I}}(R)_{R}\right)$, where $P \cong e\left(\oplus^{n} R_{R}\right)$, for some $n$ and $e=e^{2} \in \operatorname{End}\left(\oplus^{n} R_{R}\right)$.

If $R$ is not semiprime, the above remark does not hold. For example, let $R=\mathbb{Z}_{3}\left[S_{3}\right]$, the group algebra of $S_{3}$ over the field $\mathbb{Z}_{3}$ of three elements, where $S_{3}$ is the symmetric group on $\{1,2,3\}$. As was shown in [15, Example 1.1], $R_{R}$ is not strongly FI-extending. Thus, $H_{\mathfrak{S F} \mathfrak{I}}\left(R_{R}\right)$ does not exist because $R_{R}$ is injective.

According to [25], $E\left(M_{R}\right)$ is called $\Sigma$-injective if $\oplus_{\Lambda} E\left(M_{R}\right)$ is injective for any non-empty set $\Lambda$. Thus $E\left(M_{R}\right)$ is $\Sigma$-injective if and only if $E\left(\oplus_{\Lambda} M_{R}\right)=\oplus_{\Lambda} E\left(M_{R}\right)$ for any non-empty set $\Lambda$.

COROLlary 7. Assume that $R$ is a semiprime right Goldie ring. Then every projective right $R$-module $P_{R}$ has the absolute FI-extending hull. Moreover, if $P \cong e\left(\oplus_{\Lambda} R_{R}\right)$ with $e=e^{2} \in \operatorname{End}_{R}\left(\oplus_{\Lambda} R_{R}\right)$, then $H_{\mathfrak{F} \mathfrak{I}}\left(P_{R}\right) \cong e\left(\oplus_{\Lambda} \widehat{Q}_{\mathfrak{F} \mathfrak{T}}(R)_{R}\right)$.

Proof. By [25, Lemma 2 and Corollary 3], if a ring $R$ is semiprime right Goldie, then $E\left(R_{R}\right)$ is $\Sigma$-injective. Now the rest of the proof follows from that of Theorem 6.

The FI-extending hull of a module, in general, is distinct from the injective hull of the module or its extending hull (if it exists). From Corollary $7, H_{\mathfrak{F} \mathfrak{I}}\left(\oplus_{\Lambda} \mathbb{Z}_{\mathbb{Z}}\right)=\oplus_{\Lambda} \mathbb{Z}_{\mathbb{Z}}$, where $\mathbb{Z}$ is the ring of integers. However in $E\left(\oplus_{\Lambda} \mathbb{Z}_{\mathbb{Z}}\right)=\oplus_{\Lambda} \mathbb{Q}_{\mathbb{Z}}$, where $\Lambda$ is infinite and $\mathbb{Q}$ is the field of rational numbers, there is not even a minimal extending essential extension of $\oplus_{\Lambda} \mathbb{Z}_{\mathbb{Z}}$. Our next result gives an alternative description of $H_{\mathfrak{F} \mathfrak{I}}\left(P_{R}\right)$ from Theorem 6 .

Proposition 8. Assume that $P_{R}$ is a finitely generated projective module over a semiprime ring $R$. Then $H_{\mathfrak{F} \mathfrak{I}}\left(P_{R}\right) \cong P \otimes_{R} \widehat{Q}_{\mathfrak{F} \mathfrak{I}}(R)$ as $\widehat{Q}_{\mathfrak{F} \mathfrak{I}}(R)$-modules. Hence $H_{\mathfrak{F} \mathfrak{I}}\left(P_{R}\right)$ is also a finitely generated projective $\widehat{Q}_{\mathfrak{F} \mathfrak{I}}(R)$-module.

Proof. The proof is routine. As in Step 3 of the proof of Theorem 6, we may assume that $P=e\left(\oplus^{n} R_{R}\right)$ with $e=e^{2} \in \operatorname{Mat}_{n}(R)$. Let $\alpha \in P \otimes_{R} \widehat{Q}_{\mathfrak{F} \mathfrak{I}}(R)$. Then there are $a_{1}, a_{2}, \ldots, a_{n}, b_{1}, b_{2}, \ldots, b_{n}, c_{1}, c_{2}, \ldots, c_{n} \in R$ and $q_{1}, q_{2}, \ldots, q_{k} \in \widehat{Q}_{\mathfrak{F} \mathfrak{I}}(R)$ such that

$$
\alpha=e\left(\begin{array}{c}
a_{1} \\
a_{2} \\
\vdots \\
a_{n}
\end{array}\right) \otimes q_{1}+e\left(\begin{array}{c}
b_{1} \\
b_{2} \\
\vdots \\
b_{n}
\end{array}\right) \otimes q_{2}+\cdots+e\left(\begin{array}{c}
c_{1} \\
c_{2} \\
\vdots \\
c_{n}
\end{array}\right) \otimes q_{k}
$$

Since $H_{\mathfrak{F} \mathfrak{I}}\left(P_{R}\right)=e\left(\oplus^{n} \widehat{Q}_{\mathfrak{F} \mathfrak{I}}(R)_{R}\right)$ by Theorem $6, \sigma: P \otimes_{R} \widehat{Q}_{\mathfrak{F} \mathfrak{I}}(R) \rightarrow H_{\mathfrak{F} \mathfrak{I}}\left(P_{R}\right)$ can be defined by

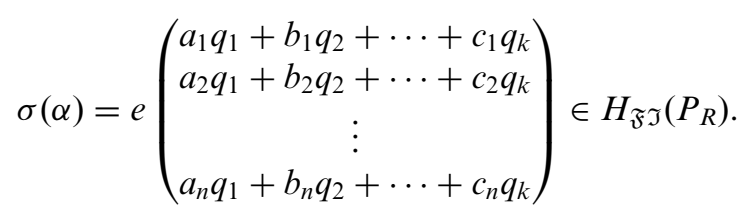

Then $\sigma$ is an onto homomorphism. To show that $\sigma$ is one-to-one, note that $\tau:\left(\oplus^{n} R_{R}\right) \otimes_{R} \widehat{Q}_{\mathfrak{F} \mathfrak{I}}(R) \cong \oplus^{n} \widehat{Q}_{\mathfrak{F} \mathfrak{I}}(R)$ by corresponding $\gamma_{1} \otimes x_{1}+\cdots+\gamma_{m} \otimes$ $x_{m} \in\left(\oplus^{n} R_{R}\right) \otimes_{R} \widehat{Q}_{\mathfrak{F} \mathfrak{I}}(R)$ (with $\gamma_{i} \in \oplus^{n} R_{R}$ and $x_{i} \in \widehat{Q}_{\mathfrak{F} \mathfrak{I}}(R)$ for $i=1,2, \ldots, m$ ) to 
$\gamma_{1} x_{1}+\gamma_{2} x_{2}+\cdots+\gamma_{m} x_{m} \in \oplus^{n} \widehat{Q}_{\mathfrak{F} \mathfrak{I}}(R)$. Since $P=e\left(\oplus^{n} R_{R}\right)$, so $P \otimes_{R} \widehat{Q}_{\mathfrak{F} \mathfrak{I}}(R)$ is a direct

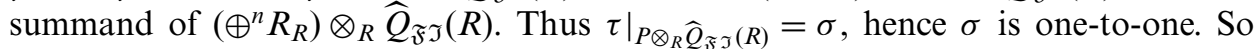
$P \otimes_{R} \widehat{Q}_{\mathfrak{F} \mathfrak{I}}(R) \cong H_{\mathfrak{F} \mathfrak{I}}\left(P_{R}\right)$. Moreover, $\sigma$ is a $\widehat{Q}_{\mathfrak{F} \mathfrak{I}}(R)$-module isomorphism.

The existence of absolute FI-extending hulls is not always guaranteed, even in the presence of non-singularity, as the next example shows.

EXAMPLE 9. There exists a right non-singular ring $R$ such that $R_{R}$ does not have the absolute FI-extending hull. Let $F$ be a field and let

$$
R=\left\{\left(\begin{array}{lll}
a & 0 & x \\
0 & a & y \\
0 & 0 & c
\end{array}\right) \mid a, x, y, c \in F\right\}
$$

Then $R$ is right non-singular with $Q(R)=\operatorname{Mat}_{3}(F)$. Assume to the contrary that there exists $H_{\mathfrak{F} \mathfrak{I}}\left(R_{R}\right)$. Let

$$
H_{1}=\left(\begin{array}{ccc}
F & 0 & F \\
0 & F & F \\
0 & 0 & F
\end{array}\right) \text { and } H_{2}=\left\{\left(\begin{array}{ccc}
a+b & a & x \\
0 & b & y \\
0 & 0 & c
\end{array}\right) \mid a, b, c, x, y \in F\right\} .
$$

Then $H_{1}$ and $H_{2}$ are right FI-extending rings (see [17, Example 3.19]). Since $H_{1}$ and $H_{2}$ are right rings of quotients of $R$, it follows that $H_{1}$ and $H_{2}$ are FI-extending right $R$-modules by [17, Proposition 1.8]. Thus $H_{\mathfrak{F} \mathfrak{I}}\left(R_{R}\right) \subseteq H_{1} \cap H_{2}=R$, so $H_{\mathfrak{F} \mathfrak{I}}\left(R_{R}\right)=R_{R}$. By [14, Corollary 1.6], $R_{R}$ is not FI-extending. Thus we have a contradiction.

EXAMPLE 10. There exists a prime Noetherian ring $R$ (hence $R=H_{\mathfrak{F} \mathfrak{I}}\left(R_{R}\right)$ ) but $H_{\mathfrak{E}}\left(R_{R}\right)$ does not exist. Let $R=\operatorname{Mat}_{2}(F[x, y])$, where $F$ is a field. Then $E\left(R_{R}\right)=$ $Q(R)=\operatorname{Mat}_{2}(F(x, y))$, where $F(x, y)$ is the field of fractions of $F[x, y]$. Note that $H_{\mathfrak{F} \mathfrak{I}}\left(R_{R}\right)=R_{R}$. Let $S=\operatorname{Mat}_{2}(F(y)[x])$ and $T=\operatorname{Mat}_{2}(F(x)[y])$. Now both $S_{S}$ and $T_{T}$ are extending. Thus by [17, Proposition 1.8] $S_{R}$ and $T_{R}$ are extending. Assume to the contrary that $H_{\mathfrak{E}}\left(R_{R}\right)$ exists. Then $H_{\mathfrak{E}}\left(R_{R}\right) \leq S_{R} \cap T_{R}$. Since $S_{R} \cap T_{R}=R_{R}$ by [19, Example 3.10], $R_{R}=H_{\mathfrak{E}}\left(R_{R}\right)$. So $R_{R}$ is extending, which is a contradiction because the domain $F[x, y]$ is not Prüfer.

LEMma 11. Assume that $R$ is a semiprime ring. Then $\widehat{Q}_{\mathfrak{F} \mathfrak{I}}(f R f)=f \widehat{Q}_{\mathfrak{F} \mathfrak{I}}(R) f$ for any $0 \neq f=f^{2} \in R$.

Proof. The proof appears in [18].

From Osofsky [34], there is a prime $\operatorname{ring} R$ with $\mathbf{J}(R)=0$ such that $E\left(R_{R}\right)$ is a nonrational extension of $R_{R}$. So $Q(R)_{R}$ is not injective, thus $\operatorname{End}\left(E\left(R_{R}\right)\right) \supsetneqq Q(R)$ as rings by [31, p. 95, Proposition 3]. Hence $Q\left(\operatorname{End}\left(R_{R}\right)\right) \neq \operatorname{End}\left(E\left(R_{R}\right)\right)$ (see also [16, Proposition 2.6]). However, a special case of our next result shows that $\widehat{Q}_{\mathfrak{F} \mathfrak{T}}(R) \cong$ $\operatorname{End}\left(H_{\mathfrak{F} \mathfrak{I}}\left(R_{R}\right)\right)$ for a semiprime ring $R$.

THEOREM 12. Assume that $R$ is a semiprime ring and $P_{R}$ is a finitely generated projective module. Then we have the following:

(i) $\widehat{Q}_{\mathfrak{F} \mathfrak{I}}\left(\operatorname{End}\left(P_{R}\right)\right) \cong \operatorname{End}\left(H_{\mathfrak{F} \mathfrak{I}}\left(P_{R}\right)\right)$ as rings.

(ii) $\operatorname{Rad}\left(H_{\mathfrak{F} \mathfrak{I}}\left(P_{R}\right)_{\widehat{Q}_{\mathfrak{F} \mathfrak{I}}(R)}\right) \cap P=\operatorname{Rad}\left(P_{R}\right)$, where $\operatorname{Rad}(-)$ is the Jacobson radical of a module.

Proof. (i) Since $P_{R} \cong e\left(\oplus^{n} R_{R}\right)$ with $e=e^{2} \in \operatorname{Mat}_{n}(R)$, it follows that $\operatorname{End}\left(P_{R}\right) \cong$ $e \operatorname{Mat}_{n}(R) e$. Also by Theorem $6, H_{\mathfrak{F} \mathfrak{I}}\left(P_{R}\right) \cong e\left(\oplus^{n} \widehat{Q}_{\mathfrak{F} \mathfrak{I}}(R)\right)$. Thus $\operatorname{End}\left(H_{\mathfrak{F} \mathfrak{I}}\left(P_{R}\right)\right) \cong$ 
$e \operatorname{Mat}_{n}\left(\operatorname{End}\left(\widehat{Q}_{\mathfrak{Y} \mathfrak{I}}(R)_{R}\right) e . \operatorname{Since} \operatorname{End}\left(\widehat{Q}_{\mathfrak{F} \mathfrak{T}}(R)_{R}\right) \cong \widehat{Q}_{\mathfrak{F} \mathfrak{I}}(R)\right.$ by Lemma $4, \operatorname{End}\left(H_{\mathfrak{F} \mathfrak{I}}\left(P_{R}\right)\right) \cong$ $e \operatorname{Mat}_{n}\left(\operatorname{End}_{R}\left(\widehat{Q}_{\mathfrak{F} \mathfrak{I}}(R)_{R}\right)\right) e \cong e \operatorname{Mat}_{n}\left(\widehat{Q}_{\mathfrak{F} \mathfrak{T}}(R)\right) e$. Now from Lemma 11, $\widehat{Q}_{\mathfrak{F} \mathfrak{T}}\left(e \operatorname{Mat}_{n}\right.$ $(R) e)=e \widehat{Q}_{\mathfrak{F} \mathfrak{J}}\left(\operatorname{Mat}_{n}(R)\right) e$ because $\operatorname{Mat}_{n}(R)$ is semiprime and $0 \neq e=e^{2} \in \operatorname{Mat}_{n}(R)$. Also by [17, Corollary 5.6] since $\operatorname{Mat}_{n}\left(\widehat{Q}_{\mathfrak{F} \mathfrak{T}}(R)\right)=\widehat{Q}_{\mathfrak{F} \mathfrak{T}}\left(\operatorname{Mat}_{n}(R)\right)$, it follows that

$$
\operatorname{End}\left(H_{\mathfrak{F} \mathfrak{I}}\left(P_{R}\right)\right) \cong e \widehat{Q}_{\mathfrak{F} \mathfrak{I}}\left(\operatorname{Mat}_{n}(R)\right) e=\widehat{Q}_{\mathfrak{F} \mathfrak{J}}\left(e \operatorname{Mat}_{n}(R) e\right) \cong \widehat{Q}_{\mathfrak{F} \mathfrak{J}}\left(\operatorname{End}\left(P_{R}\right)\right)
$$

(ii) Let $\kappa: P_{R} \rightarrow e\left(\oplus^{n} R_{R}\right)$ be an isomorphism, where $e=e^{2} \in \operatorname{Mat}_{n}(R)$. Then there exists an isomorphism $\bar{\kappa}: H_{\mathfrak{F} \mathfrak{I}}\left(P_{R}\right) \rightarrow e\left(\oplus^{n} \widehat{Q}_{\mathfrak{F} \mathfrak{J}}(R)_{R}\right)$ which is an extension of $\kappa$. Thus to show that $\operatorname{Rad}\left(H_{\mathfrak{F} \mathfrak{I}}\left(P_{R}\right) \widehat{Q}_{\mathfrak{F} \mathfrak{I}(R)}\right) \cap P=\operatorname{Rad}\left(P_{R}\right)$, it is enough to see that $\operatorname{Rad}\left(e\left(\oplus^{n} \widehat{Q}_{\mathfrak{F} \mathfrak{T}}(R)_{\left.\widehat{Q}_{\mathfrak{F} \mathfrak{J}(R)}\right)}\right) e\left(\oplus^{n} R_{R}\right)=\operatorname{Rad}\left(e\left(\oplus^{n} R_{R}\right)\right)\right.$.

Let $M_{R}=e\left(\oplus^{n} R_{R}\right)$. Since $M_{R}$ is projective, we have that $\operatorname{Rad}\left(M_{R}\right)=$ $M \mathbf{J}(R)=e\left(\oplus^{n} R_{R}\right) \mathbf{J}(R)=e\left(\oplus^{n} \mathbf{J}(R)\right)$. Now $H_{\mathfrak{F} \mathfrak{T}}\left(M_{R}\right)=e\left(\oplus^{n} \widehat{Q}_{\mathfrak{F} \mathfrak{I}}(R)\right)$ by Theorem 6. Therefore, $H_{\mathfrak{F} \mathfrak{I}}\left(M_{R}\right)$ is a projective right $\widehat{Q}_{\mathfrak{F} \mathfrak{I}}(R)$-module, so it follows that $\operatorname{Rad}\left(H_{\mathfrak{F} \mathfrak{I}}\left(M_{R}\right)_{\widehat{Q}_{\mathfrak{F}}(R)}\right)=\left(H_{\mathfrak{F} \mathfrak{I}}(M)_{\widehat{Q}_{\mathfrak{F} \mathfrak{J}}(R)}\right) \mathbf{J}\left(\widehat{Q}_{\mathfrak{F} \mathfrak{I}}(R)\right)=e\left(\oplus^{n} \widehat{Q}_{\mathfrak{F} \mathfrak{I}}(R)\right) \mathbf{J}\left(\widehat{Q}_{\mathfrak{F} \mathfrak{I}}(R)\right)=$ $e\left(\oplus^{n} \mathbf{J}\left(\widehat{Q}_{\mathfrak{F} \mathfrak{I}}(R)\right)\right.$. Say $e\left(\alpha_{1}+\alpha_{2}+\cdots+\alpha_{n}\right) \in \operatorname{Rad}\left(H_{\mathfrak{F} \mathfrak{J}}\left(M_{R}\right)_{\widehat{Q}_{\mathfrak{F}}(R)}\right) \cap M$ with $\alpha_{i} \in$ $\mathbf{J}\left(\widehat{Q}_{\mathfrak{F} \mathfrak{I}}(R)\right)$. Then $e\left(\alpha_{1}+\alpha_{2}+\cdots+\alpha_{n}\right)=e\left(r_{1}+r_{2}+\cdots+r_{n}\right)$ for some $r_{i} \in R$. Let $e=\left(a_{i j}\right) \in \operatorname{Mat}_{n}(R)$. Then

$$
\left(a_{i j}\right)\left(\begin{array}{c}
\alpha_{1} \\
\alpha_{2} \\
\vdots \\
\alpha_{n}
\end{array}\right)=\left(a_{i j}\right)\left(\begin{array}{c}
r_{1} \\
r_{2} \\
\vdots \\
r_{n}
\end{array}\right) \text {, }
$$

so $a_{i 1} \alpha_{1}+a_{i 2} \alpha_{2}+\cdots+a_{i n} \alpha_{n}=a_{i 1} r_{1}+a_{i 2} r_{2}+\cdots+a_{i n} r_{n}$ for $i=1,2, \ldots, n$. Since $\alpha_{1}, \alpha_{2}, \ldots, \alpha_{n} \in \mathbf{J}\left(\widehat{Q}_{\mathfrak{F} \mathfrak{I}}(R)\right)$, it follows that $a_{i 1} \alpha_{1}+a_{i 2} \alpha_{2}+\cdots+a_{i n} \alpha_{n} \in \mathbf{J}\left(\widehat{Q}_{\mathfrak{F} \mathfrak{I}}(R)\right)$ for $i=1,2, \ldots, n$. Thus $a_{i 1} \alpha_{1}+a_{i 2} \alpha_{2}+\cdots+a_{i n} \alpha_{n}=a_{i 1} r_{1}+a_{i 2} r_{2}+\cdots+a_{i n} r_{n} \in$ $\mathbf{J}\left(\widehat{Q}_{\mathfrak{F} \mathfrak{I}}(R)\right) \cap R$ for $i=1,2, \ldots, n$. By [19, Theorem 2.2], $\mathbf{J}\left(\widehat{Q}_{\mathfrak{F} \mathfrak{I}}(R)\right) \cap R=\mathbf{J}(R)$, hence $a_{i 1} \alpha_{1}+a_{i 2} \alpha_{2}+\cdots+a_{i n} \alpha_{n}=a_{i 1} r_{1}+a_{i 2} r_{2}+\cdots+a_{i n} r_{n} \in \mathbf{J}(R)$. Thus

$$
e\left(\begin{array}{c}
r_{1} \\
r_{2} \\
\vdots \\
r_{n}
\end{array}\right)=\left(\begin{array}{c}
a_{11} r_{1}+\cdots+a_{1 n} r_{n} \\
a_{21} r_{1}+\cdots+a_{2 n} r_{n} \\
\vdots \\
a_{n 1} r_{1}+\cdots+a_{n n} r_{n}
\end{array}\right) \in e\left(\begin{array}{c}
\mathbf{J}(R) \\
\mathbf{J}(R) \\
\vdots \\
\mathbf{J}(R)
\end{array}\right)=e\left(\oplus^{n} R_{R}\right) \mathbf{J}(R)=\operatorname{Rad}\left(M_{R}\right) .
$$

Therefore, $\operatorname{Rad}\left(H_{\mathfrak{F} \mathfrak{I}}\left(M_{R}\right)_{\widehat{Q}_{\mathfrak{Y} \mathfrak{J}(R)}}\right) \cap M \subseteq \operatorname{Rad}\left(M_{R}\right)$.

On the other hand, note that $\mathbf{J}\left(\widehat{Q}_{\mathfrak{F} \mathfrak{I}}(R)\right) \cap R=\mathbf{J}(R)$ again by $[\mathbf{1 9}$, Theorem 2.2]. Thus it follows that $\operatorname{Rad}\left(M_{R}\right)=e\left(\oplus^{n} \mathbf{J}(R)\right) \subseteq e\left(\oplus^{n} \mathbf{J}\left(\widehat{Q}_{\mathfrak{F} \mathfrak{I}}(R)\right)\right)=$ $\operatorname{Rad}\left(H_{\mathfrak{F} \mathfrak{I}}\left(M_{R}\right)_{\widehat{Q}_{\mathfrak{F}}(R)}\right), \quad$ hence $\quad \operatorname{Rad}\left(M_{R}\right) \subseteq \operatorname{Rad}\left(H_{\mathfrak{F} \mathfrak{T}}\left(M_{R}\right) \widehat{Q}_{\mathfrak{F} \mathfrak{\jmath}}(R) \cap M\right.$. So $\operatorname{Rad}\left(H_{\mathfrak{F} \mathfrak{J}}\left(M_{R}\right)_{\widehat{Q}_{\mathfrak{F} \mathfrak{J}}(R)}\right) \cap M=\operatorname{Rad}\left(M_{R}\right) . \quad$ Therefore, $\quad \operatorname{Rad}\left(H_{\mathfrak{F} \mathfrak{J}}\left(P_{R}\right)_{\widehat{Q}_{\mathfrak{F} \mathfrak{J}}(R)}\right) \cap P=$ $\operatorname{Rad}\left(P_{R}\right)$.

When $P_{R}$ is a progenerator, we have the following:

COROLlary 13. Let $R$ be a semiprime ring.

(i) If $P_{R}$ is a progenerator of the category Mod-R, then $H_{\mathfrak{F} \mathfrak{J}}\left(P_{R}\right)_{\widehat{Q}_{\mathfrak{F} \mathfrak{J}}(R)}$ is a progenerator of the category Mod- $\widehat{Q}_{\mathfrak{F} \mathfrak{T}}(R)$.

(ii) If $R$ and $S$ are Morita equivalent, then $\widehat{Q}_{\mathfrak{F} \mathfrak{I}}(R)$ and $\widehat{Q}_{\mathfrak{F} \mathfrak{T}}(S)$ are Morita equivalent. 
Proof. (i) Assume that $P_{R}$ is a progenerator for Mod-R. Let $P_{R} \cong e\left(\oplus^{n} R_{R}\right)$ with $e=e^{2} \in \operatorname{Mat}_{n}(R)$ and let $S=\operatorname{End}\left(P_{R}\right)$. Then $R$ is Morita equivalent to $S$ and $S \cong e \operatorname{Mat}_{n}(R) e$ with $\operatorname{Mat}_{n}(R) e \operatorname{Mat}_{n}(R)=\operatorname{Mat}_{n}(R)$. Now $\widehat{Q}_{\mathfrak{F} \mathfrak{I}}(S) \cong$ $e \operatorname{Mat}_{n}\left(\widehat{Q}_{\mathfrak{F} \mathfrak{I}}(R)\right) e$ by Lemmas 3 and 11 since $S$ is semiprime. Moreover, we have that $\operatorname{Mat}_{n}\left(\widehat{Q}_{\mathfrak{F} \mathfrak{I}}(R)\right) e \operatorname{Mat}_{n}\left(\widehat{Q}_{\mathfrak{F} \mathfrak{I}}(R)\right)=\operatorname{Mat}_{n}\left(R \mathbf{B}(Q(R)) e \operatorname{Mat}_{n}(R \mathbf{B}(Q(R))=\right.$ $\left[\operatorname{Mat}_{n}(R) e \operatorname{Mat}_{n}(R)\right] \mathbf{B}(Q(R))=\operatorname{Mat}_{n}(R) \mathbf{B}(Q(R))=\operatorname{Mat}_{n}(R \mathbf{B}(Q(R)))=\operatorname{Mat}_{n}\left(\widehat{Q}_{\mathfrak{F} \mathfrak{I}}(R)\right)$ by noting that $\widehat{Q}_{\mathfrak{F} \mathfrak{I}}(R)=R \mathbf{B}(Q(R))$ from Lemma 3 . Also by Theorem 12(i), it follows that $\widehat{Q}_{\mathfrak{F I}}(S) \cong \widehat{Q}_{\mathfrak{F I}}\left(\operatorname{End}\left(P_{R}\right)\right) \cong \operatorname{End}\left(H_{\mathfrak{F} \mathfrak{I}}\left(P_{R}\right)_{R}\right)=e \operatorname{Mat}_{n}\left(\operatorname{End}\left(\widehat{Q}_{\mathfrak{F} \mathfrak{I}}(R)_{R}\right)\right) e \cong$ $e \operatorname{Mat}_{n}\left(\widehat{Q}_{\mathfrak{F} \mathfrak{I}}(R)\right) e=\operatorname{End}\left(H_{\mathfrak{F} \mathfrak{I}}\left(P_{R}\right)_{\widehat{Q}_{\mathfrak{F} \mathfrak{I}}(R)}\right)$. Thus we have that $H_{\mathfrak{F} \mathfrak{I}}\left(P_{R}\right)_{\widehat{Q}_{\mathfrak{F} \mathfrak{I}}(R)}$ is a progenerator of the category $\operatorname{Mod}-\widehat{Q}_{\mathfrak{F} \mathfrak{I}}(R)$.

(ii) This part was proved in [18]. We include a proof for the sake of completeness. Suppose that $R$ and $S$ are Morita equivalent. Then there exists a progenerator $P_{R}$ of the category Mod- $R$ such that $S=\operatorname{End}\left(P_{R}\right)$. Thus by Theorem 12(i), $\widehat{Q}_{\mathfrak{F} \mathfrak{I}}(S) \cong \operatorname{End}\left(H_{\mathfrak{F} \mathfrak{I}}\left(P_{R}\right)_{R}\right)=\operatorname{End}\left(H_{\mathfrak{F} \mathfrak{I}}\left(P_{R}\right)_{\widehat{Q}_{\mathfrak{F} \mathfrak{I}}(R)}\right)$, so $\widehat{Q}_{\mathfrak{F} \mathfrak{I}}(R)$ and $\widehat{Q}_{\mathfrak{F} \mathfrak{I}}(S)$ are Morita equivalent.

We notice that the converse of Corollary 13(ii) is not true. Let $R=\mathbb{Z}[G]$ the group ring of $G$ over $\mathbb{Z}$, where $G$ is the group of order two. Then $R$ is semiprime by [31, p. 162, Proposition 8]. Let $S=\widehat{Q}_{\mathfrak{F} \mathfrak{I}}(R)$. From Lemma 3 and the fact that $Q(R)=Q(S)$, it follows that $\widehat{Q}_{\mathfrak{F} \mathfrak{I}}(R)=\widehat{Q}_{\mathfrak{F} \mathfrak{I}}(S)$. If $R$ and $S$ are Morita equivalent, then $R \cong S$ since $R$ and $S$ are commutative (see [30, p. 494, Corollary 18.42]). So we have a contradiction because $R$ is not quasi-Baer by [13, Example 1.11].

Recall from [37] that a module $M_{R}$ is a quasi-Baer module if for any $N_{R} \unlhd M_{R}$, there exists $h=h^{2} \in \Lambda=\operatorname{End}\left(M_{R}\right)$ such that $\ell_{\Lambda}(N)=\Lambda h$, where $\ell_{\Lambda}(N)=\{\lambda \in \Lambda \mid$ $\lambda N=0\}$. It is clear that $R_{R}$ is a quasi-Baer module if and only if $R$ is a quasi-Baer ring. Also it is shown in [37] that $M_{R}$ is quasi-Baer if and only if for any $I \unlhd \Lambda$ there exists $g=g^{2} \in \Lambda$ such that $r_{M}(I)=g M$, where $r_{M}(I)=\{m \in M \mid I m=0\}$. Moreover, if $M_{R}$ is quasi-Baer, then $\operatorname{End}\left(M_{R}\right)$ is a quasi-Baer ring [37, Theorem 4.1]. Close connections between quasi-Baer modules and FI-extending modules are investigated in [37].

In the next result, we obtain another close connection between FI-extending modules and quasi-Baer modules which also generalizes some of the equivalences in [12, Theorem 4.7].

THEOREM 14. Assume that $P_{R}$ is a finitely generated projective module over a semiprime ring $R$. Then the following are equivalent:

(i) $P_{R}$ is (strongly) FI-extending.

(ii) $P_{R}$ is quasi-Baer.

(iii) $\operatorname{End}\left(P_{R}\right)$ is a quasi-Baer ring.

(iv) $\operatorname{End}\left(P_{R}\right)$ is a right FI-extending ring.

Proof. Let $P_{R} \cong e\left(\oplus^{n} R_{R}\right)$ with $e=e^{2} \in \operatorname{End}\left(\oplus^{n} R_{R}\right) \cong \operatorname{Mat}_{n}(R)$ and $n>0$.

(i) $\Rightarrow$ (ii) Assume that $P_{R}$ is FI-extending. Then $P_{R}=H_{\mathfrak{F} \mathfrak{T}}\left(P_{R}\right) \cong e\left(\oplus^{n} \widehat{Q}_{\mathfrak{q} \mathfrak{B}}(R)_{R}\right)$ by Theorem 6 and Lemma 3 . Since $\operatorname{End}\left(\widehat{Q}_{\mathfrak{q} \mathfrak{B}}(R)_{R}\right) \cong \widehat{Q}_{\mathfrak{q} \mathfrak{B}}(R)$ by Lemma 4 , we see that $\widehat{Q}_{\mathfrak{q} \mathfrak{B}}(R)_{R}$ is quasi-Baer. So $\oplus^{n} \widehat{Q}_{\mathfrak{q} \mathfrak{B}}(R)_{R}$ is quasi-Baer [37, Proposition 3.19]. Hence $e\left(\oplus^{n} \widehat{Q}_{\mathfrak{q} \mathfrak{B}}(R)_{R}\right)$ is quasi-Baer from [37, Theorem 3.17]. Therefore $P_{R}$ is quasi-Baer.

(ii) $\Rightarrow$ (iii) It follows from [37, Theorem 4.1].

(iii) $\Rightarrow$ (i) Suppose that $\operatorname{End}\left(P_{R}\right)$ is a quasi-Baer ring. Then $\operatorname{End}\left(P_{R}\right) \cong$ $e \operatorname{Mat}_{n}(R) e$ is quasi-Baer. Now $\widehat{Q}_{\mathfrak{q} \mathfrak{B}}\left(\operatorname{Mat}_{n}(R)\right)=\operatorname{Mat}_{n}(R) \mathbf{B}\left(Q\left(\operatorname{Mat}_{n}(R)\right)=\right.$ $\operatorname{Mat}_{n}(R) \mathbf{B}\left(\operatorname{Mat}_{n}(Q(R))\right)=\operatorname{Mat}_{n}(R) \mathbf{B}(Q(R))=\operatorname{Mat}_{n}(R \mathbf{B}(Q(R)))=\operatorname{Mat}_{n}\left(\widehat{Q}_{\mathfrak{q} \mathfrak{B}}(R)\right)$ by Lemma 3. Thus $e \operatorname{Mat}_{n}(R) e=\widehat{Q}_{\mathfrak{q} \mathfrak{B}}\left(e \operatorname{Mat}_{n}(R) e\right)=e \widehat{Q}_{\mathfrak{q} \mathfrak{B}}\left(\operatorname{Mat}_{n}(R)\right) e=e \operatorname{Mat}_{n}$ 
$\left(\widehat{Q}_{\mathfrak{q} \mathfrak{B}}(R)\right) e$ by Lemma 11 . Let $f \in \mathbf{B}(Q(R))=\mathbf{B}\left(\operatorname{End}\left(E\left(R_{R}\right)\right)\right)$ and let $I_{n}$ be the identity matrix in $\operatorname{Mat}_{n}(R)$. Then we have that $f I_{n} \in \mathbf{B}\left(\operatorname{Mat}_{n}(Q(R))\right)=$ $\mathbf{B}\left(\operatorname{End}\left(\oplus^{n} E\left(R_{R}\right)\right)\right)$. Thus $e \cdot f I_{n} \cdot e \in e \operatorname{Mat}_{n}\left(\widehat{Q}_{\mathfrak{q} \mathfrak{B}}(R)\right) e=e \operatorname{Mat}_{n}(R) e$. Let $e \cdot f I_{n} \cdot e=$ $\left(\alpha_{i j}\right) \in e \operatorname{Mat}_{n}(R) e \subseteq \operatorname{Mat}_{n}(R)$. Then we see that

$$
e\left(\begin{array}{c}
f R \\
\vdots \\
f R
\end{array}\right)=e \cdot f I_{n}\left(\begin{array}{c}
R \\
\vdots \\
R
\end{array}\right)=e \cdot f I_{n} \cdot e\left(\begin{array}{c}
R \\
\vdots \\
R
\end{array}\right)=e\left(\alpha_{i j}\right) e\left(\begin{array}{c}
R \\
\vdots \\
R
\end{array}\right) \subseteq e\left(\begin{array}{c}
R \\
\vdots \\
R
\end{array}\right)
$$

Hence $e\left(\oplus^{n} \widehat{Q}_{\mathfrak{q} \mathfrak{B}}(R)_{R}\right)=e\left(\oplus^{n} R_{R}\right)$ because $\widehat{Q}_{\mathfrak{q} \mathfrak{B}}(R)=R \mathbf{B}(Q(R))$ by Lemma 3 . Hence from Theorem 6, $H_{\mathfrak{F} \mathfrak{I}}\left(e\left(\oplus^{n} R_{R}\right)\right)=e\left(\oplus^{n} R_{R}\right)$, so $e\left(\oplus^{n} R_{R}\right)$ is (strongly) FI-extending. Therefore $P_{R}$ is (strongly) FI-extending.

(iii) $\Leftrightarrow$ (iv) Since $\operatorname{End}\left(P_{R}\right)$ is semiprime, this equivalence follows from [12, Theorem 4.7].

For a ring $R$, let $\operatorname{Aut}(R)$ denote the group of ring automorphisms of $R$. Let $G$ be a subgroup of $\operatorname{Aut}(R)$. For $r \in R$ and $g \in G$ let $r^{g}$ denote the image of $r$ under $g$. We use $R^{G}$ to denote the fixed ring of $R$ under $G$ (i.e. $R^{G}=\left\{r \in R \mid r^{g}=r\right.$ for every $\left.g \in G\right\}$ ). The skew group ring, $R * G$, is defined to be $R * G=\oplus \sum_{g \in G} R g$ with addition given componentwise and multiplication given as follows: if $a, b \in R$ and $g, h \in G$, then $(a g)(b h)=a b^{g^{-1}} g h \in R g h$.

Let $R$ be a semiprime ring. For $g \in \operatorname{Aut}(R)$, let $\phi_{g}=\left\{x \in Q^{m}(R) \mid x r^{g}=\right.$ $r x$ for each $r \in R\}$, where $Q^{m}(R)$ is the Martindale right ring of quotients of $R$. We say that $g$ is $X$-outer if $\phi_{g}=0$. A subgroup $G$ of $\operatorname{Aut}(R)$ is called $X$-outer on $R$ if every $1 \neq g \in G$ is X-outer (see [5, pp. 139-143]).

Assume that $G$ is a finite group of ring automorphisms of a $\operatorname{ring} R$. Say $G=$ $\left\{g_{1}, \ldots, g_{n}\right\}$. For $r \in R$ and $\alpha=a_{1} g_{1}+\cdots+a_{n} g_{n} \in R * G$ with $a_{i} \in R$, define $r \cdot \alpha=$ $r^{g_{1}} a_{1}^{g_{1}}+\cdots+r^{g_{n}} a_{n}^{g_{n}}$. Then $R$ is a right $R * G$-module. Moreover, we see that $R^{G} R_{R * G}$ is an $\left(R^{G}, R * G\right)$-bimodule. Also End $\left(R_{R * G}\right) \cong R^{G}$.

If $A$ is a $C^{*}$-algebra (not necessarily unital), then the set $\mathcal{F}$ of all norm closed essential two-sided ideals forms a filter directed downward by inclusion. The ring $Q_{b}(A)$ denotes the algebraic inductive limit of $\{M(I)\}_{I \in \mathcal{F}}$, where $M(I)$ is the multiplier $C^{*}$-algebra of $I$. In [3], the ring $Q_{b}(A)$ is called the symmetric normed algebra of quotients of $A$. The norm completion of $Q_{b}(A)$, i.e. the $C^{*}$-algebra inductive limit $M_{\text {loc }}(A)$ of $\{M(I)\}_{I \in \mathcal{F}}$, is called the local multiplier algebra of $A$ which was used to solve operator equations on $A$ (see [24] and [35]). In [1-4], $Q_{b}(A)$ and $M_{\text {loc }}(A)$ of a $C^{*}$-algebra $A$ have been extensively studied. For more details on local multiplier algebras, see [5].

According to [5, Definition 3.2.1, p. 73], for a $C^{*}$-algebra $A$, the $C^{*}$-subalgebra $\overline{A C_{b}(A)}$ (i.e., the norm closure of $A C_{b}(A)$ in $\left.M_{\mathrm{loc}}(A)\right)$ of $M_{\mathrm{loc}}(A)$ is called the bounded central closure of $A$ and denoted by ${ }^{c} A$, where $C_{b}(A)$ is the centre $\operatorname{Cen}\left(Q_{b}(A)\right)$ of $Q_{b}(A)$. If $A={ }^{c} A$, then $A$ is called boundedly centrally closed. It is shown in [5, Theorem 3.2.8 and Corollary 3.2.9, pp. 75-76] that the local multiplier algebra and the bounded central closure of a $C^{*}$-algebra are boundedly centrally closed. Also, it is shown in [19, Lemma 4.12] that a unital $C^{*}$-algebra $A$ is boundedly centrally closed if and only if $A$ is quasi-Baer.

By [5] a $*$-preserving ring automorphism of a $C^{*}$-algebra is called an $*$-automorphism. When $A$ is a unital $C^{*}$-algebra with a finite group $G$ of $*$-automorphisms of $A$, it was shown in [5, Section 4.4, pp. 139-141] that $A * G$ and $A^{G}$ are $C^{*}$-algebras. 
COROLlary 15. Let A be a unital $C^{*}$-algebra and $G$ a finite group of $*$-automorphisms of $A$. Then the following conditions (i), (ii) and (iii) are equivalent:

(i) $A_{A * G}$ is (strongly) FI-extending.

(ii) $A_{A * G}$ is quasi-Baer.

(iii) $A^{G}$ is a boundedly centrally closed $C^{*}$-algebra.

Further, if $G$ is $X$-outer, then the following conditions (iv) and ( $v)$ are equivalent to conditions ( $i)-($ iii):

(iv) $A * G$ is a boundedly centrally closed $C^{*}$-algebra.

(v) $A$ is G-quasi-Baer (i.e. the right annihilator of a $G$-invariant ideal of $A$ is generated by an idempotent).

Proof. Recall that End $\left(A_{A * G}\right) \cong A^{G}$. Since $|G|$ is invertible, $e=|G|^{-1}\left(\sum_{g \in G} g\right) \in$ $A * G$ is an idempotent and $A_{A * G} \cong e(A * G)_{A * G}$ as $A * G$-modules. Thus $A_{A * G}$ is a finitely generated projective module. Thus by [19, Lemma 4.12] $A^{G}$ is quasi-Baer if and only if $A^{G}$ is boundedly centrally closed. Hence the equivalence of (i)-(iii) follows immediately from Theorem 14.

Further, assume that $G$ is X-outer. Note that the condition (iv) is equivalent to the fact that $A * G$ is quasi-Baer. Hence from [19, Lemma 4.12] and [27, Theorem 10], (iii), (iv) and (v) are equivalent.

Note that in [37, Example 4.2], there is an example of a module $M_{R}$ such that $\operatorname{End}\left(M_{R}\right)$ is a quasi-Baer ring, but $M_{R}$ is not quasi-Baer. In this paper we have shown that for $\mathfrak{M}=\mathfrak{F} \mathfrak{I}$, if $R$ is a semiprime ring then $H_{\mathfrak{F} \mathfrak{I}}\left(R_{R}\right)=\widehat{Q}_{\mathfrak{F} \mathfrak{I}}(R)$. This motivates the following problem:

Problem. For a given class $\mathfrak{M}$ of modules, determine necessary and/or sufficient conditions on $R$ such that $H_{\mathfrak{M}}\left(R_{R}\right)=\widehat{Q}_{\mathfrak{M}}(R)$.

ACKNOWLEDGEMENTS. The authors thank the referee for his/her helpful suggestions and comments for improving the paper. Also the authors are grateful for the support they received from the Mathematics Research Institute, Columbus and for the kind hospitality and support of Busan National University, the Ohio State University at Lima, and the University of Louisiana at Lafayette.

\section{REFERENCES}

1. P. Ara, The extended centroid of $C^{*}$-algebras Arch. Math. 54 (1990), 358-364.

2. P. Ara, On the symmetric algebra of quotients of a $C^{*}$-algebra, Glasgow Math. J. 32 (1990), 377-379.

3. P. Ara and M. Mathieu, A local version of the Dauns-Hofmann theorem, Math. Z. 208 (1991), 349-353.

4. P. Ara and M. Mathieu, An application of local multipliers to centralizing mappings of $C^{*}$-algebras, Quart. J. Math. Oxford 44 (1993), 129-138. 2003).

5. P. Ara and M. Mathieu, Local multipliers of $C^{*}$-algebras (Springer, Berlin, Germany,

6. E. A. Azoff, Kaplansky-Hilbert modules and the self-adjointness of operator algebras, Am. J. Math. 100 (1978), 957-972.

7. G. F. Birkenmeier, A generalization of FPF rings, Comm. Algebra 17 (1989), 855-884. $319-326$.

8. G. F. Birkenmeier, Decomposition of Baer-like rings, Acta Math. Hungar. 59 (1992),

9. G. F. Birkenmeier, When does a supernilpotent radical essentially split-off? J. Algebra 172 (1995), 49-60. 
10. G. F. Birkenmeier, G. Călugăreanu, L. Fuchs and H. P. Goeters, The fully invariant extending property for Abelian groups, Comm. Algebra 29 (2001), 673-685.

11. G. F. Birkenmeier, J. Y. Kim and J. K. Park, On quasi-Baer rings, in Algebra and its applications (Huynh D. V., Jain S. K. and López-Permouth S. R., Editors)(Amer. Math. Soc., Providence, RI, 2000), 67-92.

12. G. F. Birkenmeier, B. J. Müller and S. T. Rizvi, Modules in which every fully invariant submodule is essential in a direct summand, Comm. Algebra 30 (2002), 1395-1415.

13. G. F. Birkenmeier and J. K. Park, Triangular matrix representations of ring extensions, J. Algebra 265 (2003), 457-477.

14. G. F. Birkenmeier, J. K. Park and S. T. Rizvi, Generalized triangular matrix rings and the fully invariant extending property, Rocky Mountain J. Math. 32 (2002), 1299-1319.

15. G. F. Birkenmeier, J. K. Park and S. T. Rizvi, Modules with fully invariant submodules essential in fully invariant summands, Comm. Algebra 30 (2002), 1833-1852.

16. G. F. Birkenmeier, J. K. Park and S. T. Rizvi, An essential extension with nonisomorphic ring structures, Algebra and its applications (Huynh D. V., Jain S. K. and López-Permouth S. R., Editors) (Amer. Math. Soc., Providence, RI, 2006), 29-48.

17. G. F. Birkenmeier, J. K. Park and S. T. Rizvi, Ring hulls and applications, J. Algebra 304 (2006), 633-665.

18. G. F. Birkenmeier, J. K. Park and S. T. Rizvi, Hulls of ring extensions, Canad. Math. Bull. (in press).

19. G. F. Birkenmeier, J. K. Park and S. T. Rizvi, Hulls of semiprime rings with applications to $C^{*}$-algebras, J. Algebra (in press).

20. D. P. Blecher and C. LeMerdy, Operator algebras and their modules - An operator space approach (Clarendon, Oxford, 2004).

21. A. W. Chatters and C. R. Hajarnavis, Rings in which every complement right ideal is a direct summand, Quart. J. Math. Oxford 28 (1977), 61-80.

22. W. E. Clark, Twisted matrix units semigroup algebras, Duke Math. J. 34 (1967), 417-424.

23. N. V. Dung, D. V. Huynh, P. F. Smith and R. Wisbauer, Extending Modules (Longman, Harlow, 1994).

24. G. A. Elliott, Automorphisms determined by multipliers on ideals of a $C^{*}$-algebra, J. Funct. Anal. 23 (1976), 1-10.

25. C. Faith, Rings with ascending condition on annihilators, Nagoya Math. J. 27 (1966), $179-191$.

26. S. Grabiner, Finitely generated, Noetherian, and Artinian Banach modules, Indiana Univ. Math. J. 26 (1977), 413-425.

27. H. L. Jin, J. Doh and J. K. Park, Group actions on quasi-Baer rings, Canad. Math. Bull. (in press).

28. I. Kaplansky, Modules over operator algebras, Am. J. Math. 75 (1953), 839-858.

29. I. Kaplansky, Rings of operators (Benjamin, New York, 1968).

30. T. Y. Lam, Lectures on Modules and Rings (Springer-Verlag, Berlin, Germany, 1998).

31. J. Lambek, Lectures on rings and modules (Chelsea, New York, 1986).

32. A. C. Mewborn, Regular rings and Baer rings, Math. Z. 121 (1971), 211-219.

33. B. J. Müller and S. T. Rizvi, Ring decompositions of CS-rings, in Abstracts for methods in module theory conference (Colorado Springs, May 1991).

34. B. L. Osofsky, A non-trivial ring with non-rational injective hull, Canad. Math. Bull. 10 (1967), 275-282.

35. G. K. Pedersen, Approximating derivations on ideals of $C^{*}$-algebras, Invent. Math. 45 (1978), 299-305.

36. A. Pollingher and A. Zaks, On Baer and quasi-Baer rings, Duke Math. J. 37 (1970), $127-138$.

37. S. T. Rizvi and C. S. Roman, Baer and quasi-Baer modules, Comm. Algebra 32 (2004), 103-123. 